\title{
Sobre a ação de Euler-Heisenberg e o espalhamento da luz pela luz
}

\author{
On the Euler-Heisenberg action and the light scattering by light \\ J. Furtado ${ }^{* 1,20}$ \\ ${ }^{1}$ Centro Brasileiro de Pesquisas Físicas, Rio de Janeiro, RJ, Brasil \\ ${ }^{2}$ Universidade Federal do Cariri, Juazeiro do Norte, CE, Brasil
}

\begin{abstract}
Recebido em 01 de Setembro, 2018. Revisado em 30 de Novembro, 2018. Aceito em 24 de Dezembro, 2018.
Este trabalho tem como objetivo apresentar uma revisão, com fins didáticos e de divulgação científica, acerca dos desenvolvimentos que envolvem o espalhamento da luz pela luz e sua relação com a ação de Euler-Heisenberg. Apresentamos inicialmente uma sucinta introdução histórica seguida por discussões sobre a ação de EulerHeisenberg em vários contextos, mas em especial dois, o método do tempo-próprio de Fock-Schwinger e a violação de simetria de Lorentz. Por fim, discutimos os resultados recentes obtidos pela colaboração ATLAS do LHC que apontam uma evidência para o espalhamento da luz pela luz.
\end{abstract}

Palavras-chave: Espalhamento da luz pela luz, ação de Euler-Heisenberg, eletrodinâmica quântica.

This work has as objective to present a review, with pedagogical and scientific divulgation purposes, about the developments on the subject of the light by light scattering and its relation with the Euler-Heisenberg effective action. We present initially a brief historical overview followed by discussion over the Euler-Heisenberg action in several contexts, but specially in two, Fock-Schwinger proper-time method and Lorentz violation symmetry. Finally we discuss the recent results obtained by the ATLAS collaboration of the LHC which point to an evidence of the light by light scattering.

Keywords: Light by light scattering, Euler-Heisenberg action, quantum eletrocynamics.

\section{Introdução}

O Modelo Padrão (MP), que descreve três das quatro interações fundamentais, a saber, as interações eletromagnética, fraca e forte, em sua versão mínima, é dotado da simetria $S U(3) \times S U(2) \times U(1)$. Apesar de ser fenomenologicamente bem sucedido, o MP deixa algumas questões em aberto, tais como o problema da hierarquia ou a falta de uma descrição quântica para a gravitação. Até outubro de 2013 a não detecção do Bóson de Higgs era tida como uma lacuna deixada pelo MP, pois a existência do bóson de Higgs era uma previsão do modelo que não havia sido detectada até então. Uma outra previsão do MP que até agosto de 2017 estava também como uma lacuna era o processo do espalhamento da luz pela luz, sendo que evidências para sua ocorrência foram detectadas pela colaboração ATLAS. Discutiremos estas evidências ao final deste trabalho e o iniciaremos com uma revisão histórica acerca do fenômeno.

Em 1928 Dirac publicara seu trabalho acerca da teoria relativística do elétron 1], que atraiu de imediato a atenção de Heisenberg para a "teoria do mar de Dirac", um novo cenário para o vácuo quântico da QED (Quantum Electrodynamics). A descoberta do pósitron em 1932 e a sua associação com essa teoria, marca, praticamente, o nascimento da QED. Heisenberg publicara em 1934 dois trabalhos 2, 3], que formalizam o tratamento das flutuações quânticas inerentes ao cenário do mar de Dirac, e logo foi percebido que estas flutuações levariam a não linearidades.

É interessante ressaltar aqui que o processo de espalhamento da luz pela luz não é previsto pela teoria eletromagnética clássica, uma vez que classicamente a luz é descrita pelas equações de Maxwell e pelo princípio da superposição. Como não há cargas e correntes no espaço vazio e fótons não possuem carga elétrica, logo, estes não interagem entre si. No cenário quântico, contudo, devido a possibilidade de criação de pares elétron-pósitron, dois fótons podem espalhar um ao outro devido a interação com as partículas virtuais (ver Fig. 1).

Dentro deste contexto, foi discutida a possibilidade de ocorrência física do espalhamento fóton-fóton (ou espalhamento da luz pela luz), mesmo quando a energia dos fótons não é suficiente para criar pares (partículaantipartícula). Hans Euler foi o primeiro a estudar o espalhamento da luz pela luz através do formalismo da matriz de densidade [4], e em 1935 um breve artigo foi publicado por Kockel e Euler apresentando o resultado para o limite de baixas frequências da amplitude de espalhamento da luz pela luz [5].

*Endereço de correspondência: job.furtado@ufca.edu.br. 


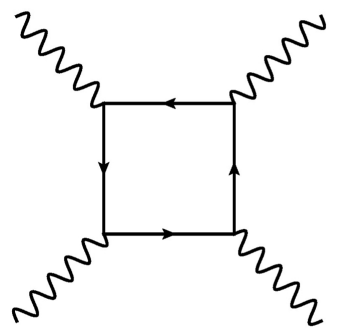

(a)

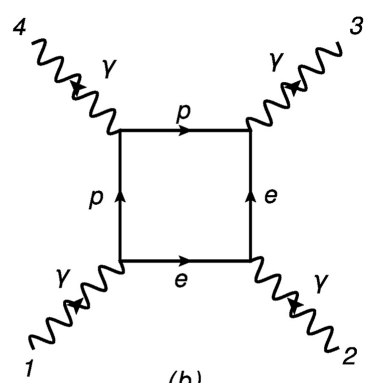

(b)
Figura 1: Gráfico de Feynman (a) que descreve o processo do espalhamento da luz pela luz. Uma das formas de se interpretar o diagrama de Feynman apresentado em (a) para o processo de espalhamento da luz pela luz é como está representado em (b). Em (b) o fóton (1) divide-se em um par elétron-pósitron virtuais, o elétron absorve o fóton (2) enquanto que o pósitron emite o fóton (4) e por fim o par elétron-pósitron se aniquila dando origem ao fóton (3).

Analogamente ao que acontece com um material dielétrico que, quando submetido à ação de um campo elétrico não tão intenso ao ponto de o "ionizar", dá origem a uma polarização induzida no material gerada pelo rearranjamento das cargas em seu interior, o trabalho de Euler e Kockel tornava claro que o vácuo quântico poderia ser visto como um meio dielétrico,uma vez que devido à presença de flutuações quânticas (criação e aniquilação de pares de partículas virtuais elétron-pósitron) o vácuo da $Q E D$ poderia ser interpretado como um meio polarizável. Eles encontraram a correção quântica não linear dominante para a lagrangiana de Maxwell, como sendo

$L=\frac{\vec{E}^{2}-\vec{B}^{2}}{2}+\frac{1}{90 \pi} \frac{\hbar c}{e^{2}} \frac{1}{E_{0}^{2}}\left[\left(\vec{E}^{2}-\vec{B}^{2}\right)^{2}+7(\vec{E} \cdot \vec{B})^{2}\right]$

em que

$$
E_{0}=\frac{e}{r_{0}^{2}}
$$

Na lagrangiana acima $\vec{E}$ e $\vec{B}$ são os campos elétrico e magnético, respectivamente. A quantidade $r_{0}=e^{2} / m c^{2} \approx$ $2,8 \times 10^{-15} \mathrm{~m}$ é o raio de um elétron clássico, enquanto que $E_{0}=e / r_{0}^{2}$ representa a "intensidade de campo no raio do elétron" (termo alcunhado por Euler e Kockel em [5]).

Euler e Kockel interpretaram a polarização do vácuo como sendo o seguinte resultado:

$$
\begin{aligned}
\vec{D} & =\vec{E}+\frac{1}{90 \pi} \frac{\hbar c}{e^{2}} \frac{1}{E_{0}^{2}} \\
& \times\left[4\left(\vec{E}^{2}-\vec{B}^{2}\right) \vec{E}+14(\vec{E} \cdot \vec{B}) \vec{B}\right] \\
\vec{H} & =\vec{B}+\frac{1}{90 \pi} \frac{\hbar c}{e^{2}} \frac{1}{E_{0}^{2}} \\
& \times\left[4\left(\vec{E}^{2}-\vec{B}^{2}\right) \vec{B}-14(\vec{E} \cdot \vec{B}) \vec{E}\right],
\end{aligned}
$$

que são obtidos a partir das seguintes relações constitutivas:

$$
\vec{D}=\frac{\partial L}{\partial \vec{E}} \quad \text { e } \vec{H}=-\frac{\partial L}{\partial \vec{B}} .
$$

Note que assim como em meios macroscópicos, em que as quantidades $\vec{D}$ e $\vec{H}$ não são simples múltiplos de $\vec{E}$ e $\vec{B}$, respectivamente, as equações (3) mostram claramente que o vácuo pode ser interpretado como um meio dielétrico (para mais detalhes ver [6]). A correção quântica não linear dominante dada à lagrangiana de Maxwell em (1) dá origem aos termos de polarização elétrica e magnética induzidos em (3).

Além disto, Euler e Kockel também discutiram a similaridade formal entre o resultado por eles obtido (eq.(1)) com àquele obtido por Born e Infeld [7], em que uma correção não linear à teoria de Maxwell fora obtida, porém, dentro de uma perspectiva clássica.

Posteriormente, em 1936, o seminal trabalho de Euler e Heisenberg [8] apresentava uma expressão fechada para a correção não linear à lagrangiana de Maxwell, isto é, uma expressão não perturbativa incorporando todas as ordens no campo eletromagnético (em uma aproximação de campo constante). A lagrangiana por eles encontrada é dada por

$$
\begin{aligned}
\mathcal{L}= & \frac{e^{2}}{h c} \int_{0}^{\infty} \frac{d \eta}{\eta^{3}} e^{-\eta}\left\{i \eta^{2}(\vec{E} \cdot \vec{B}) \frac{[\cos \Lambda+c \cdot c]}{[\cos \Lambda-c \cdot c]}+\right. \\
& \left.+\varepsilon_{c}^{2}+\frac{\eta^{2}}{3}\left(\vec{B}^{2}-\vec{E}^{2}\right)\right\},
\end{aligned}
$$

com $\Lambda$ sendo

$$
\Lambda=\left(\frac{\eta}{\varepsilon_{c}} \sqrt{\vec{E}^{2}-\vec{B}^{2}+2 i(\vec{E} \cdot \vec{B})}\right) .
$$

O resultado completo não perturbativo apresentado na equação acima permitiu a Heisenberg ter um vislumbre precoce, através da análise das divergências logarítmicas, da renormalização da carga elétrica. A representação da equação (5) é conhecida hoje em dia como "forma do tempo próprio", em que o parâmetro $\eta$ é identificado como o tempo próprio (atualmente utiliza-se $s$ ou $\tau$ para identificar o tempo próprio) e $\varepsilon_{c}$ é uma intensidade crítica de campo $\left(\varepsilon_{c}=\alpha E_{0}\right)$ da ordem de $10^{16} \mathrm{~V} / \mathrm{cm}$ atualmente conhecida como limite de Schwinger, que marca o início da região em que os efeitos não lineares passam a ser considerados. Note ainda que se a lagrangiana (5) for expandida em uma aproximação de campo fraco até quarta ordem, a lagrangiana (1) é recuperada. Em uma linguagem mais moderna, Euler e Kockel estudaram a polarização do vácuo da QED em uma aproximação de campo constante, obtendo as correções não lineares em termos de potências das intensidades dos campos.

Então, desde a sua descoberta, a ação efetiva de EulerHeisenberg $(\mathrm{EH})$ tem sido estudada em vários contextos, tais como o espalhamento da luz pela luz [9], relação com funções beta 10 14], procura por áxions [15], extensões não abelianas da ação de Euler-Heisenberg 16 18], 
método da função zeta 19 21], campos magnéticos intensos 22 25], produção de pares no vácuo [8, 26, 27], divisão de fótons 28 30, birrefringência no vácuo [31], ação efetiva em modelos de gravidade e teoria de cordas 32 38], dentre outros. Uma revisão mais completa acerca dos contextos em que a ação efetiva de $\mathrm{EH}$ foi extensivamente estudada pode ser vista em 39,40].

Um dos tópicos que merece atenção especial é a birrefringência no vácuo, estudada inicialmente em 1970 por Bialynicka-Birula e Bialynicki-Birula 28] A birrefringência no vácuo surge como uma consequência imediata das flutuações quânticas, que induzem no vácuo um comportamento análogo ao de um meio polarizável, como descrito pelas equações (3). Desta forma, foi mostrado em [28] que na presença de um campo eletromagnético intenso, o índice de refração para a propagação da luz é maior que um $(n>1)$ e depende da polarização do feixe. Sendo o índice de refração $n>1$, a velocidade de propagação da luz é sutilmente reduzida na presença de um campo intenso. Além disso, a dependência do índice de refração com a polarização do feixe promove uma diferença na velocidade de propagação da luz para polarização paralela e perpendicular ao campo. Para o caso de um campo magnético intenso, por exemplo, os índices de refração para as polarizações perpendicular e paralela são, respectivamente,

$$
\begin{aligned}
& n_{\perp}=1+\frac{2 \alpha}{45 \pi}\left(\frac{B_{0}}{B_{c}}\right)^{2} \\
& n_{\|}=1+\frac{7 \alpha}{90 \pi}\left(\frac{B_{0}}{B_{c}}\right)^{2},
\end{aligned}
$$

sendo $B_{0}$ a intensidade do campo magnético e $B_{c}=\varepsilon_{c} / c$ o campo magnético crítico de Schwinger. Vê-se então que $n_{\|}>n_{\perp}$. Para mais detalhes ver 41 .

Mais recentemente, estudos acerca da indução da lagrangiana de EH em uma linguagem e notação mais moderna foram realizados 42, bem como estudos para além de um loop 43 44 (após os trabalhos iniciais realizados por Ritus 45 ). Os desenvolvimentos para além de um loop foram realizados primordialmente usando o formalismo do mundo linha (worldline formalism) e análise de Borel na QED em $(1+1)$-dimensões.

A lagrangiana de EH também recebeu atenção na interface entre a teoria quântica de campos e a física da matéria condensada, devido aos trabalhos de Horava [46], que propuseram sistemas com uma anisotropia de escala entre espaço e tempo, tornando possível o estudo da lagrangiana de Euler-Heisenberg e a produção de pares de Schwinger em uma bicamada de grafeno [4]. A anisotropia de escala sugerida por Horava com o intuito de construir uma teoria quântica para a gravitação livre de divergências ultravioletas mostrou-se extremamente apropriada para a descrição de férmions em uma bicamada de grafeno. Diferentemente do que acontece com férmions em uma única camada de grafeno, que comportam-se obdecendo uma relação de dispersão relativística $(E \propto p)$ e uma invariância de escala $\vec{r} \rightarrow b \vec{r}, t \rightarrow b t$, férmions em uma bicamada de grafeno obedecem uma relação de dispersão tipo Schrödinger $\left(E \propto p^{2}\right)$ e um escalonamento anisotrópico do tipo $\vec{r} \rightarrow b \vec{r}, t \rightarrow b^{2} t$, que é precisamente o caso $z=2$ no modelo proposto por Horava, sendo $z$ o expoente crítico que controla o grau de anisotropia. Dentro deste contexto foi discutido em 47. a taxa de produção de pares de Schwinger (fenômeno descrito pela ação de Euler-Heisenberg) em uma bicamada de grafeno, sempre em comparação com o caso de uma única camada de grafeno, que é invariante de escala.

No cenário de violação de simetria de Lorentz, os estudos acerca da ação efetiva de EH foram considerados, em sua grande maioria, no contexto de divisão de fótons [48 54], principalmente após o trabalho publicado por Kostelecky e Pickering [50] que afirma a possibilidade de ocorrência do fenômeno da divisão tripla de fótons no vácuo, um processo que é descrito pela ação efetiva de $\mathrm{EH}$ mas que na QED usual não ocorre. Mais recentemente foi discutida a influência do setor não mínimo da extensão do modelo padrão com violação de simetria de Lorentz no processo de espalhamento da luz pelz luz [55]. Desta forma, em vista dos desenvolvimentos recentes no que diz respeito a ação de EH no cenário de violação de simetria de Lorentz, discutiremos este cenário com mais detalhes ao longo do trabalho.

A geração radiativa da ação de EH é de suma importância, visto que, as correções não lineares apresentadas tornam possíveis os cálculos para amplitudes de espalhamentos específicos, tais como o espalhamento de um fóton em um campo eletromagnético de um núcleo (espalhamento Delbrück) [56 |57. e o espalhamento de um fóton em um campo magnético intenso [58,59. Recentemente vinha-se sendo discutido que o espalhamento fóton-fóton poderia ser observado no LHC (Large Hadron Collider) 60]. Como discutiremos ao final deste trabalho, evidências experimentais desta detecção foram observadas pela colaboração ATLAS.

Após este sucinto resumo histórico o trabalho dividiráse em seções que versarão acerca do desenvolvimento da formulação do tempo-próprio (seção II), o contexto de violação de simetria de Lorentz (seção III) e a recente detecção do espalhamento fóton-fóton (seção IV).

\section{Formulação do tempo próprio}

O conceito de tempo próprio, introduzido pela primeira vez por Minkowski em 1908, é definido como o tempo marcado por um relógio ao longo de um intervalo tipo tempo. Definido desta forma o tempo próprio é, portanto, independente do sistema de coordenadas e um escalar de Lorentz. A ideia do tempo próprio serviu inicialmente para estabeler as bases da teoria da relatividade especial e foi extendida de maneira bastante direta para a relatividade geral. Dentro do ponto de vista experimental, o tempo próprio é o que é medido experimentalmente, enquanto que o tempo em algum sistema de coordenadas específico é calculado a partir do tempo próprio. 
A extensão da formulação do tempo próprio para a mecânica quântica relativística se deu de uma maneira não muito imediata. Em 1937 Fock 62 publica um trabalho em que ele apresenta uma formulação da equação de Dirac em termos da evolução do tempo próprio, enquanto que em 1946 Stückelberg [63], reinterpretando as soluções de energia negativa da equação de Dirac, propõe um cenário físico em que pósitrons propagam-se para trás no tempo. Tais trabalhos não receberam atenção da comunidade científica até os posteriores trabalho de Feynman e Schwinger 64 67.

Os trabalhos publicados por Feynman tentavam extender a formulação de integral de trajetória, já bem estabelecida para a mecânica quântica não relativística, para a teoria relativística do pósitron de Dirac. Feynman propôs então representar um elemento da matrix de transição como uma integral de trajetória sobre todos os caminhos no espaço-tempo, com os caminhos sendo parametrizados através de um quinto parâmetro. Nesta construção Feynman notou que haviam trajetórias que aparentemente voltavam no tempo, mas motivado pelo trabalho de Stückelberg ele identificou as trajetórias que evoluiam para frente no tempo com os elétrons (partículas), enquanto que as evoluiam para trás no tempo foram identificadas com pósitrons (antipartículas). Sobre o trabalho de Feynman, Nambu diz que "o tempo em si perde seu sentido enquanto indicador do desenvolvimento dos fenômenos", tal afirmativa fundamenta-se no fato de que, na interpretação de Stückelberg-Feynman, partículas movimentam-se para frente no tempo enquanto que antipartículas movimentam-se para trás no tempo.

$\mathrm{Na}$ interpretação convencional da teoria quântica de campos, antipartículas existem como entidades distintas das suas partículas correspondentes e portanto evoluem para frente no tempo, como qualquer partícula. Já na interpretação de Stückelberg-Feynman, antipartículas não existem enquanto entidades ontologicamente distintas das suas correspondentes partículas, uma vez que as antipartículas correspondem simplesmente as suas partículas evoluindo para trás no tempo. Neste cenário não há criação e aniquilação de partículas, mas sim uma mudança na direção de evolução temporal das partículas, do passado para o futuro e vice-versa. Na Figura 2, por exemplo, vê-se uma representação do diagrama de Feynman para o processo de espalhamento elétron-pósitron, ou espalhamento Bhabha. Na figura o tempo está ao longo da horizontal, crescendo da esquerda para a direita. $\mathrm{Na}$ interpretação de Stückelberg-Feynman o elétron não é destruído, ele simplesmente muda sua direção de propagação no tempo ao emitir um fóton. Este elétron que agora propaga-se para trás no tempo exibe o comportamento de um pósitron que evolui para frente no tempo. As interpretações acima mencionadas são atualmente aceitas como equivalentes e portanto não há violação de causalidade na interpretação de Stückelberg-Feynman.

Logo após o trabalho de Feynman, em 1951, os resultados originais de Euler e Heisenberg são reformulados por

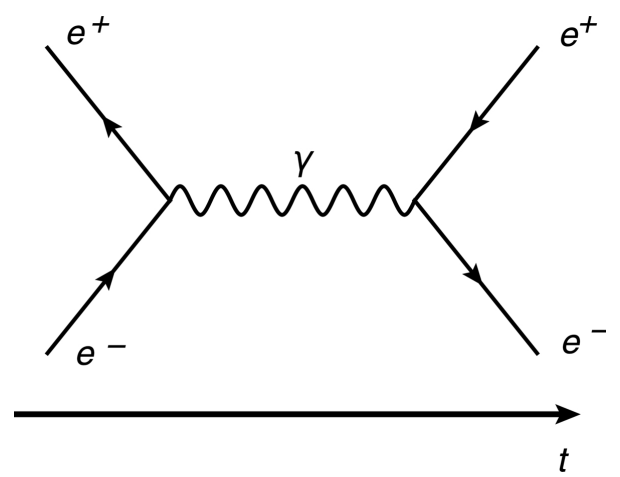

Figura 2: Diagrama de Feynman do processo de espalhamento Bhabha (elétron-pósitron)

Schwinger 67] e apresentados em uma nova linguagem, a eletrodinâmica quântica renormalizada. O método apresentado por Schwinger interpreta o tempo próprio como um regulador da invariância de gauge, provendo assim uma nova maneira de efetuar cálculos sem que a invariância de gauge seja perdida durante o procedimento. O método desenvolvido por Schwinger apresenta o resultado exato da ação efetiva para dois casos especiais, a saber, o caso com o campo constante e o caso com o campo tipo onda plana. Para tal Schwinger escreve a lagrangiana efetiva em termos do operador de evolução do tempo próprio $U(s)$, de modo que,

$$
\mathcal{L}(x)=\frac{i}{2} \int_{0}^{\infty} \frac{d s}{s} \exp \left(-i m^{2} s\right) \operatorname{tr}\langle x|U(s)| x\rangle
$$

sendo

$$
U(s)=\exp (-i \mathcal{H} s) \text { e } \mathcal{H}=\Pi_{\mu}^{2}-\frac{e}{2} \sigma_{\mu \nu} F^{\mu \nu} .
$$

Aqui, $\Pi_{\mu}=p_{\mu}-e A_{\mu}$ representa o quadrimomento enquanto que o tensor $\sigma_{\mu \nu}$ é definido em termos do anticomutador das matrizes de Dirac como $\sigma_{\mu \nu}=(i / 2)\left[\gamma_{\mu}, \gamma_{\nu}\right]$.

Então, usando resultados já derivados por Fock e Nambu, Schwinger apresenta uma expressão completa, não perturbativa, de uma lagrangiana efetiva que apresenta correções não lineares à eletrodinâmica de Maxwell em acordo com os resultados obtidos anteriormente por Euler e Heisenberg. O trabalho de Schwinger põe o resultado encontrado por Euler e Heisenberg em uma base teórica e computacional sólida, além de ter servido de inspiração para uma série de trabalhos posteriores, inclusive em modelos com violação de simetria de Lorentz [68], que abordaremos a seguir.

\section{Violação de simetria de Lorentz}

Nesta seção discutiremos um modelo específico da chamada "física além do modelo padrão", a extensão com violação de simetria de Lorentz e CPT proposta por Kostelecky e Colladay. Como já mencionado na introdução, este cenário recebeu bastante atenção na literatura nos 
últimos anos e dentro do contexto da ação de EH isso se deu, em especial, devido a publicação de um artigo pelo próprio Kostelecky que apresenta um resultado não nulo para tripla divisão de fótons no vácuo. Este fenômeno, descrito pelo diagrama apresentado na Figura 1a, não ocorre na QED usual, o que faria da divisão tripla de fótons no vácuo um efeito inerente do cenário de violação de simetria de Lorentz.

No contexto do espalhamento da luz pela luz, sendo este um efeito permitido na QED usual, a influência dos termos extra (como veremos adiante) que violam as simetrias de Lorentz e CPT à lagrangiana da QED contribuem com pequenos desvios na seção de choque do processo. Estes pequenos desvios tornariam-se perceptíveis somente em regimes de mais altas energias, uma vez que tais contribuições são oriundas de uma extensão do modelo padrão convencional, podendo inclusive serem suprimidos pela massa de Planck. Recentemente foi argumentado [55] que sinais indicativos de violação de simetria de Lorentz no processo de espalhamento da luz pela luz possam ser mais facilmente detectados nas futuras observações do LHC em setores não mínimos, em detrimento do setor mínimo. Sendo assim, abordaremos de maneira mais detalhada o cenário de violação de simetria de Lorentz.

O conceito de simetria é uma das ferramentas mais poderosas no estudo da física teórica, uma vez que tornouse evidente que praticamente todas as leis da natureza estão relacionadas às simetrias. Na matemática, a generalização da ideia geométrica de simetria culminou no desenvolvimento da teoria de grupos. Na física, o conceito de simetria encontra-se manifesto explicitamente no teorema de Noether, que afirma, de maneira simplificada, que a cada simetria contínua está associada uma quantidade conservada.

Contudo, só em meados da década de 70, modelos físicos que incorporavam violação de simetrias passaram a ser efetivamente estudados. Na física de altas energias, o trabalho seminal de P. Higgs [69] apresenta o processo de quebra espontânea de simetria, em que um campo escalar adquire um valor esperado no vácuo não nulo, provendo massa para as partículas do modelo padrão. $\mathrm{Na}$ física da matéria condensada, os estudos de P.W. Anderson e Landau 70.71 mostraram que a violação de simetria é essencial para a compreensão de fenômenos, tais como a supercondutividade e cristais líquidos.

Como já dito anteriormente o MP não é tido como uma teoria completa, primordialmente devido a falta de uma descrição quântica para a gravitação. Portanto, acredita-se que o MP seja o limite de baixas energias de uma teoria mais fundamental, teoria esta que unificaria o MP e a interação gravitacional, fornecendo assim, um modelo de gravitação quântica.

A escala natural para uma teoria fundamental que incorpora a gravidade é governada pela massa de Planck $M_{\text {planck }} \approx 10^{19} \mathrm{GeV}$, que é cerca de dezessete ordens de magnitude maior que a escala eletrofraca $m_{W}$ associada ao MP. Isto sugere que, sinais experimentais observáveis emergindo a partir de uma teoria fundamental esperase que sejam suprimidos por alguma potência de $r \approx$ $m_{W} / M_{\text {Planck }} \approx 10^{-17}$.

Uma das teorias fundamentais mais promissoras ao fornecimento de uma descrição quântica da gravitação é a teoria de cordas. Contudo, devido à impossibilidade de uma verificação experimental direta da teoria, alguns modelos foram propostos a fim de estudar efeitos mensuráveis de baixas energias.

Um destes modelos, hoje conhecido como Modelo Padrão Estendido (MPE), foi inicialmente vislumbrado em 1989 através do trabalho de Kostelecky e Samuel [72], em que foi visto que interações em teorias de cordas poderiam levar a quebra espontânea de simetria de Lorentz. Estudos posteriores mostraram que teorias não comutativas e modelos de brana também levavam à quebra da invariância de Lorentz. Em 1997 e 1998 dois trabalhos publicados por Kostelecký e Colladay 73,74 finalmente deram origem ao setor mínimo do MPE em espaços-tempo planos, e em $2004 \sqrt[75]{ }$ foi publicada a extensão para espaçostempo curvos. O MPE consiste, basicamente, de uma extensão do MP que inclui em sua lagrangiana todos os possíveis termos que violam as simetrias de Lorentz e CPT.

O processo de quebra espontânea de simetria, em teorias mais fundamentais, promove o surgimento natural do efeito de violação da invariância de Lorentz e CPT 72,76 . Tal processo é análogo ao mecanismo de Higgs, contudo, no caso de um campo tensorial que contém índices de Lorentz, um valor esperado não nulo seleciona uma direção preferencial no espaço-tempo, quebrando assim, espontaneamente, a transformação de Lorentz de partícula, ao passo que a transformação de Lorentz de observador permanece inalterada. Foi baseado neste fato que Kostelecký e Colladay 73,74 formularam o modelo padrão estendido, isto é, com base na quebra espontânea de simetria de Lorentz em teorias mais fundamentais. Para mais detalhes acerca de como a presença de campos constantes quebram a equivalência entre as transformações de Lorentz de observador (transformação passiva na presença de um campo de fundo) e partícula (transformação ativa na presença de um campo de fundo) ver [77.

A quebra dinâmica da invariância de Lorentz e CPT pode ser entendida como um efeito mensurável de baixas energias que emergem de teorias como cordas 72,76 , 78 80 ou teorias de campo não comutativas [81 84$]$. O trabalho 85 foi um dos precursores no que diz respeito aos estudos acerca dos modelos que englobam violação de simetria de Lorentz, através da constatação de que o termo de Chern-Simons em $(3+1)$ dimensões é invariante de gauge, mas não de Lorentz, devido ao acoplamento do tensor eletromagnético dual a um quadrivetor constante. Contudo, o MPE é atualmente uma das teorias mais bem sucedidas no que se trata de violação da invariância de Lorentz. É importante notar que, apesar de o MPE conter termos responsáveis pela violação de simetria de 
Lorentz e de CPT, a simetria $S U(3) \times S U(2) \times U(1)$ é mantida, conservando várias propriedades importantes, assim como a renormalizabilidade da teoria [86].

Nesta seção nos deteremos ao estudo do setor mínimo da QED estendida, uma vez que espera-se que as contribuições emergentes de tais termos sejam dominantes. Muito embora, o setor não mínimo do MPE vem recebendo bastante atenção na literatura 87 90], apesar de possuir somente termos não renormalizáveis. A lagrangiana que descreve o setor mínimo da QED estendida é dada por

$$
\begin{aligned}
& \mathcal{L}=-\frac{1}{4} F_{\mu \nu} F^{\mu \nu}+\frac{1}{2} \overbrace{\left(k_{A F}\right)^{\mu}}^{M} \epsilon_{\mu \nu \lambda \rho} \overbrace{A^{\nu} F^{\lambda \rho}}^{M^{3}} \\
& -\frac{1}{4}\left(k_{F}\right)_{\mu \nu \lambda \rho} \overbrace{F^{\mu \nu} F^{\lambda \rho}}^{M^{4}}+i \bar{\psi} \Gamma^{\mu} D_{\mu} \psi-\bar{\psi} M \psi,
\end{aligned}
$$

onde $\Gamma^{\mu}=\gamma^{\mu}+\Gamma_{1}^{\mu}$ e $M=m+M_{1}$, sendo

$$
\begin{aligned}
\Gamma_{1}^{\mu} & =c^{\mu \nu} \gamma_{\nu}+d^{\mu \nu} \gamma_{5} \gamma_{\nu}+e^{\mu}+i f^{\mu} \gamma_{5}+\frac{1}{2} g^{\lambda \nu \mu} \sigma_{\lambda \nu} \\
M_{1} & =a_{\mu} \gamma^{\mu}+b_{\mu} \gamma_{5} \gamma^{\mu}+\frac{1}{2} H_{\mu \nu} \sigma^{\mu \nu} .
\end{aligned}
$$

No setor bosônico, o primeiro termo é a lagrangiana de Maxwell usual, e os outros dois termos são os que violam a simetria de Lorentz, lembrando que $F_{\mu \nu}=\partial_{\mu} A_{\nu}-\partial_{\nu} A_{\mu}$ é o tensor intensidade de campo eletromagnético e que $D_{\mu}=\partial_{\mu}+i e A_{\mu}$ é a derivada covariante. No setor fermiônico, os termos $\Gamma_{1}^{\mu}$ e $M_{1}$ são extensões (adimensionais e com dimensão de massa) da matriz $\gamma^{\mu}$ e da massa, respectivamente, que contém todas os possíveis termos que violam as simetrias de Lorentz e CPT. Todos os termos extra serão discutidos com mais detalhes a seguir.

A primeira das contribuições extra é o termo de ChernSimons quadridimensional, que é CPT ímpar e portanto viola as simetrias de Lorentz e CPT. Note que o coeficiente $\left(k_{A F}\right)^{\mu}$ tem dimensão de massa $d=1$, ao passo que o operador $A^{\nu} F^{\lambda \rho}$ (campos e derivadas) tem dimensão de massa $d=3$, visto que a lagrangiana tem dimensão de massa $d=4$. Esse termo foi bastante estudado na literatura devido sua importância tanto em sistemas de teoria quântica de campos como em sistemas de física da matéria condensada. Em particular, o termo de Chern-Simons ganhou muita atenção em $(2+1)$ dimensões, devido a sua relação com efeitos planares, tais como o efeito Hall quântico e superconditividade. Em teoria quântica de campos, alguns estudos importantes foram realizados, como por exemplo, em efeitos de dispersão e em indução radiativa 85,91. O segundo dos termos extra contém um operador com dimensão de massa $d=4$ e portanto o coeficiente $\left(k_{F}\right)_{\mu \nu \lambda \rho}$ é adimensional, além de ter simetria de tensor de Riemann.

No setor fermiônico, os operadores contraídos com os coeficientes $a_{\mu}, b_{\mu}, e_{\mu}, f_{\mu}$ e $g_{\lambda \nu \mu}$, violam as simetrias de Lorentz e CPT (CPT ímpar), enquanto que os operadores relacionados com os coeficientes $c_{\mu \nu}, d_{\mu \nu}$ e $H_{\mu \nu}$, violam apenas a simetria de Lorentz (CPT par). Contudo, sob uma certa redefinição espinorial, os termos relacionados com os coeficientes $a_{\mu}, e_{\mu}$ e $f_{\mu}$ são removidos da lagrangiana, apenas os coeficientes $\bar{c}_{\mu \nu}, \bar{d}_{\mu \nu}$ (totalmente simétrico), $\tilde{g}_{\lambda \nu \mu}$ (totalmente antissímetrico), $b_{\mu}$ e $H_{\mu \nu}$ sobrevivem. Note que os coeficientes contidos em $M_{1}$ têm dimensão de massa $d=1$, enquanto que os coeficientes contidos em $\Gamma_{1}^{\mu}$ são adimensionais.

Ainda no setor fermiônico, vale a pena enfatizar que apenas os coeficientes $b_{\mu}$ e $c_{\mu \nu}$ geram correções quânticas no setor bosônico, tal que

$$
\begin{aligned}
\left(k_{A F}\right)_{\mu} & \propto b_{\mu} \\
\left(k_{F}\right)_{\mu \nu \lambda \rho} & \propto g_{\mu \lambda} c_{\nu \rho}+g_{\nu \rho} c_{\mu \lambda}-g_{\mu \rho} c_{\nu \lambda} \\
& -g_{\nu \lambda} c_{\mu \rho} .
\end{aligned}
$$

Até o presente momento, muitos limites experimentais vêm sendo atribuídos aos coeficientes do MPE, associados a dados experimentais envolvendo oscilação de neutrinos [92 94], káons 95, 96], prótons e nêutrons 97 99], elétrons [100 103], múons [104,105 e fótons [106 109. Para uma relação mais completa, veja 110.

Em 2003 Kostelecky e Pickering mostraram que o diagrama da Figura 1, quando calculado perturbativamente levando em consideração todas as inserções nos propagadores e os novos vértices, dão origem a um resultado não nulo para a amplitude de espalhamento do processo de decaimento triplo de fótons no vácuo, indicando que, na presença de violação de simetria de Lorentz é prevista a ocorrencia de tal fenômeno, enquanto que na eletrodinâmica quântica usual tal fenômeno não é permitido. No entanto, tal resultado foi obtido através de um procedimento não convencional, que consiste em permutar as pernas externas de fótons sem carregar os índices de Lorentz, durante o cálculo de todas as possibilidades de permutação.

Contudo, alguns trabalhos posteriores [53,54 mostraram que a amplitude de espalhamento para tal processo pode dar um resultado nulo se procedimentos convencionais forem utilizados para calcular tal processo. Levando a crer que, mesmo na presença de violação de simetria de Lorentz, tal processo não é permitido. Processos associados aos gráficos que apresentam um loop de férmions com quatro pernas externas de fótons são todos descritos pela ação efetiva de Euler-Heisenberg, que foi analiticamente obtida em [54], no contexto de violação de simetria de Lorentz, para um dos termos do setor CPT-par $\left(c^{\mu \nu}\right)$ do modelo padrão estendido.

A grande importância acerca da possível ocorrência do fenômeno da divisão tripla de fótons no vácuo é que, sendo este um fenômeno que seria inerente ao cenário de violação de simetria de Lorentz, como a quebra de simetria de Lorentz emerge naturalmente a partir da quebra espontânea de simetria em teorias mais fundamentais (como já mencionado), a detecção da divisão tripla de fótons no vácuo poderia ser interpretada como uma evidência indireta da existência de teorias mais fundamentais. 


\section{Resultados experimentais}

Em agosto de 2017 foi publicado um artigo 111 da colaboração ATLAS (sigla inglesa para A Toroidal LHC ApparatuS), um dos detectores que operam no LHC, que aponta uma evidência para o espalhamento da luz pela luz como um subproduto de um processo de colisão de íons pesados. Este processo consiste basicamente de uma colisão ultraperiférica em que os parâmetros de impacto são maiores que dobro do raio do núcleo atômico em questão, de modo que, para tais eventos a interação forte pode ser inteiramente negligenciada. As intensidades dos campos eletromagnéticos para íons relativísticos são proporcionais ao número atômico Z. Para o caso do experimento em questão utilizou-se núcleos de chumbo $(\mathrm{Pb})$, que possui um número atômico $Z=82$, produzindo assim campos da ordem de $10^{25} \mathrm{Vm}^{-1}$. Campos com esta ordem de magnitude estão muito acima do limite encontrado por Schwinger a partir do qual as correções quânticas para a QED passam a ser importantes [67]. Em uma colisão ultraperiférica como a realizada (Figura 3), a interação entre os campos eletromagnéticos intensos pode ser descrita pela aproximação de fótons equivalentes [112], de modo que tais campos podem ser tratados como um feixe de fótons quase-reais. Assim, a seção de choque para a reação $P b+P b(\gamma \gamma) \rightarrow P b+P b(\gamma \gamma)$ pode ser calculada por convolução para o processo elementar $\gamma \gamma \rightarrow \gamma \gamma$. O resultado encontrado pela colaboração ATLAS possui uma significância estatística de $4.4 \sigma$.

Este foi o primeiro indício experimental que aponta para o evento do espalhamento da luz pela luz previsto por Euler e Heisenberg em 1936, muito embora detecções indiretas tenham sido realizadas a fim de encontrar correções no valor da constante de estrutura fina [113]. É importante notar ainda que o espalhamento elástico da luz pela luz ainda não foi observado. Este espalhamento elástico pode ser realizado pela colisão fóton-fóton direta em um colisor [114], pois mesmo os experimentos realizados com laser ultraintensos não foram capazes de atingir energia suficiente para evidenciar o fenômeno [115]. Além do fato de que a colisão fóton-fóton direta evidenciaria o espalhamento da luz pela luz com fótons reais, alguns autores afirmam 114 que tal processo produziria resultados com uma significância estatística maior que o valor que categoriza uma descoberta, isto é, $\sigma>5$.

Como já mencionado anteriormente o espalhamento da luz pela luz é descrito pela ação de Euler-Heisenberg (5). Outros fenômenos, também descritos pela ação de Euler-Heisenberg já foram detectados experimentalmente, tais como o espalhamento Delbrück [56 57], a divisão de fótons $\sqrt{116}$, dentre outros. O espalhamento Delbrück, representado no diagrama de Feynman da esquerda na Figura 3, ocorre quando um fóton interage fortemente com um campo elétrico muito intenso (em geral produzido nas imediações de um núcleo atômico de um átomo pesado). Na Figura 3, os traços ondulados representam os fótons enquanto que as linhas retas representam férmions virtuais. $\mathrm{O} X$ presente na perna do fóton é utilizado para indicar um campo elétrico ou magnético muito intenso.

Um processo análogo pode ocorrer quando um fóton interage com um campo muito intenso e divide-se em dois. Este processo, conhecido como divisão de fótons, está representado pelo diagrama de Feynman central na Figura 3. Mais comumente o processo da divisão de fótons $(\gamma \rightarrow \gamma \gamma)$ é discutido mediante interação de um fóton com um campo magnético muito intenso (como o campo produzido nas imediações de um magnetar). O diagrama de Feynman central na Figura 2 mostra um fóton incidente interagindo via férmions virtuais com um campo magnético muito intenso (marcado pela perna de fóton com um X) e dividindo-se em dois fótons de energia menor, mas de modo que a conservação de energia e momento seja garantida. Poderia-se de imediato pensar que o processor de divisão de fótons poderia ser descrito por um diagrama como o apresentado na Figura 4, contudo tal diagrama não é permitido devido ao teorema de Furry, que é uma consequência direta da simetria de conjugação de carga (para mais detalhes, ver [117]).

Os primeiros resultados experimentais acerca da divisão de fótons foram obtidos em 1995-1996 no colisor VEPP-4M no instituto Budker de física nuclear (Rússia). Tais resultados foram coletados a partir de uma coli-
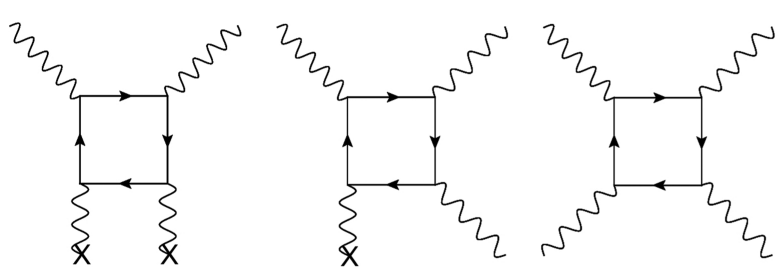
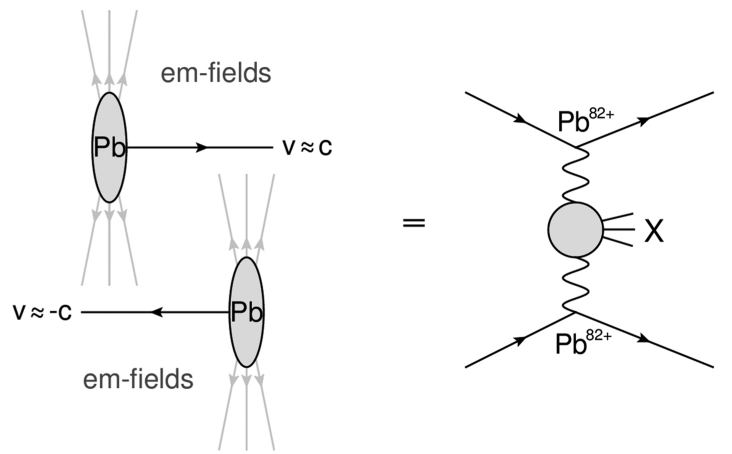

Figura 3: Gráficos de Feynman representando os três tipos de processos básicos descritos pela ação de Euler-Heisenberg, a saber, o espalhamento Delbrück (gráfico da esquerda), a divisão de fótons (gráfico do meio) e o espalhamento da luz pela luz (gráfico da direita). Bem como uma representação do processos de colisão ultraperiférica realizado no LHC. (Fonte: Referência 111]) 


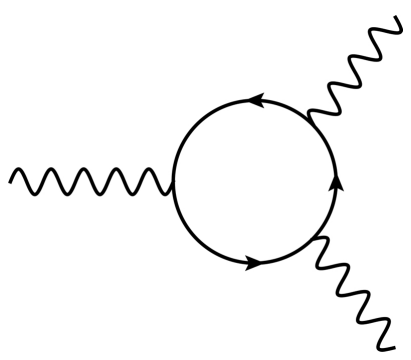

Figura 4: Diagrama de Feynman do processo de divisão de fótons não permitido pelo teorema de Furry

são entre um feixe de fótons (cerca de $1,6 \times 10^{9}$ fótons incidentes) e um alvo de germanato de bismuto. Além disso, cerca de $4 \times 10^{8}$ fótons que não atingiram o alvo foram utilizados como medida de controle. Os primeiros resultados experimentais para o espalhamento Delbrück foram obtidos também pela equipe do colisor VEPP-4M, utilizando o mesmo aparato experimental. Os resultados obtidos possuiam uma significância estatística $\sigma>5$, categorizando o resultado como uma descoberta.

\section{Conclusão}

Neste artigo buscamos apresentar inicialmente uma sucinta revisão histórica acerca dos eventos que levaram Werner Heisenberg e seu aluno Hans Euler a considerar a possibilidade da ocorrência física do fenômeno do espalhamento da luz pela luz como uma consequência das não linearidades introduzidas na eletrodinâmica de Maxwell devido à descoberta do pósitron. Discutimos também como, a partir deste fenômeno, eles foram levados à uma lagrangiana completa que descreve a propagação de fótons em campos eletromagnéticos que variam muito lentamente.

Em seguida apresentamos a sequência de eventos que levou Schwinger à formulação do método do tempo próprio, um procedimento que interpreta o tempo próprio como um regulador da invariância de gauge, provendo assim uma nova maneira de efetuar cálculos sem que a invariância de gauge seja perdida durante o processo. E como, através deste método, Schwinger foi levado à escrever uma lagrangiana que incorpora correções não lineares à eletrodinâmica de Maxwell, em acordo com os resultados já anteriormente obtidos por Euler e Heisenberg.

Introduzimos também alguns dos aspectos fundamentais acerca de uma extensão do modelo padrão da física de partículas que inclui em sua lagrangiana todos os possíveis termos que violam as simetrias de Lorentz e CPT. Discorremos também sobre como a inclusão destes termos extra à lagrangiana do modelo padrão originou controvérsias acerca da possibilidade da ocorrência do fenômeno da divisão tripla de fótons no vácuo, um fenômeno também descrito pela ação de Euler-Heisenberg.
Por fim, abordamos de maneira breve e simplificada o recente resultado obtido pela colaboração ATLAS que aponta evidências para a ocorrência do espalhamento da luz pela luz através de uma colisão de íons pesados. Bem como mencionamos também os resultados experimentais para outros processos descritos pela ação de Euler-Heisenberg, a saber, o espalhamento Delbrück e a divisão de fótons.

Este trabalho visa ser uma breve introdução ao tema já há muito tempo discutido na literatura, mas pouco presente em livros-texto (salvo algumas exceções, como [41], 117] e [118]), em especial a nível de graduação. Sendo assim tentamos abordar aspectos teóricos sem deixar de lado a fenomenologia, para o leitor interessado em aspectos gerais, enquanto que aquele interessado em aspectos mais técnicos poderá ver as referências citadas.

\section{Agradecimentos}

Gostaria de agradecer ao professor J.A. Helayël-Neto bem como aos revisores do artigo pelas valorosas sugestões e comentários.

\section{Referências}

[1] P.A.M. Dirac, Proc. Roy. Soc. Lond. A 117, 610 (1928).

[2] W. Heisenberg, Schsiche Akad. Wissen 86, 317 (1934).

[3] W. Heisenberg, Z. Phys. 90, 209 (1934).

[4] H. Euler., Annalen Phys. 26, 398 (1936).

[5] H. Euler e B. Kockel, Naturwiss 23, 246 (1935).

[6] J.D. Jackson, Classical Electrodynamics (John Wiley, Hoboken, 1999), $3^{\text {a }}$ ed.

[7] M. Born e L. Infeld, Proc. Roy. Soc. Lond. A 144, 425 (1934).

[8] W. Heisenberg e H. Euler, Z. Phys. 98, 714 (1936).

[9] R. Karplus e M. Neuman, Phys. Rev. 83, 776 (1951).

[10] V.F. Weisskopf, Phys. Rev. 56, 72 (1939).

[11] H. Pagels e E. Tomboulis, Nucl. Phys. B 143, 485 (1978).

[12] K. Fujikawa, Phys. Rev. D 48, 3922 (1993).

[13] J. Grundberg e T.H. Hansson, Annals Phys. 242, 413 (1995).

[14] M. A. Shifman e A. I. Vainshtein, Sov. J. Nucl. Phys. 44, 321 (1986).

[15] H. Gies, Eur. Phys. J. D 55, 311 (2009).

[16] M.R. Brown e M.J. Duff, Phys. Rev. D 11, 2124 (1975).

[17] M.J. Duff e M. Ramon-Medrano, Phys. Rev. D 12, 3357 (1975).

[18] V.A. Novikov, M.A. Shifman, A.I. Vainshtein e V.I. Zakharov, Fortsch. Phys. 32, 585 (1984).

[19] S.W. Hawking, Commun. Math. Phys. 55, 133 (1977).

[20] W. Dittrich, J. Phys. A 9, 1171 (1976).

[21] W. Dittrich e M. Reuter, Phys. Lett. B 128, 321 (1983).

[22] D. Kharzeev, Phys. Lett. B 633, 260 (2006)

[23] K. Fukushima, D.E. Kharzeev e H.J. Warringa, Phys. Rev. D 78, 074033 (2008)

[24] B.I. Abelev, M.M. Aggarwal, Z. Ahammed, A.V. Alakhverdyants, B.D. Anderson, D. Arkhipkin, G.S. Averichev, J. Balewski, O. Barannikova, L.S. Barnby et al., Phys. Rev. Lett. 103, 251601 (2009) 
[25] V.P. Gusynin, V.A. Miransky e I.A. Shovkovy, Phys. Rev. D 52, 4747 (1995)

[26] F. Sauter, Z. Phys. 69, 742 (1931).

[27] L.V. Keldysh, Sov. Phys. JETP 201307 (1965).

[28] Z. Bialynicka-Birula e I. Bialynicki-Birula, Phys. Rev. D 10, 2341 (1970).

[29] S.L. Adler, Annals Phys. 67, 599 (1971).

[30] S.L. Adler e C. Schubert, Phys. Rev. Lett. 77, 1695 (1996).

[31] W.Y. Tsai e T. Erber, Phys. Rev. D 12, 1132 (1975).

[32] B.S. DeWitt, Phys. Rev. 160, 1113 (1967).

[33] B.S. DeWitt, Phys. Rev. 162, 1195 (1967);

[34] B.S. DeWitt, Phys. Rev. 162, 1239 (1967).

[35] Y.B. Zeldovich e A.A. Starobinsky, Sov. Phys. JETP 34, 1159 (1972)

[36] E.S. Fradkin e A.A. Tseytlin, Nucl. Phys. B 261, 1 (1985).

[37] E.S. Fradkin e A.A. Tseytlin, Phys. Lett. B 160, 69 (1985).

[38] E.S. Fradkin e A.A. Tseytlin, Phys. Lett. B 163, 123 (1985).

[39] G.V. Dunne, Int. J. Mod. Phys. A 27, 1260004 (2012)

[40] G.V. Dunne, ArXiv:hep-th/0406216 (2004).

[41] W. Greiner e J. Reinhardt, Quantum Electrodynamics (Springer, Berlim, 2003), $3^{\text {a }}$ ed.

[42] J.S.N. Furtado e G.R. Silva, Mod. Phys. Lett. A 31, 1650153 (2016).

[43] Y. Huet, M.R. Traubenberg e C. Schubert, International Journal of Modern Physics: Conference Series Vol. 14, 383 (2012).

[44] I. Huet, D.G.C. McKeon e C. Schubert, Journal of High Energy Physics 12, 36 (2010).

[45] V.I. Ritus, Sov. Phys. JETP 42, 774 (1975).

[46] P. Horava, Phys. Rev. D 79, 084008 (2009).

[47] M.I. Katsnelson e G.E. Volovik, JETP Letters 95, 411 (2012).

[48] T. Jacobson, S. Liberati e D. Mattingly, Phys. Rev. D 67, 124011 (2003).

[49] C. Adam e F.R. Klinkhamer, Nucl. Phys. B 657, 214 (2003).

[50] V.A. Kostelecky e A.G.M. Pickering, Phys. Rev. Lett. 91, 031801 (2003).

[51] G. Gelmini, S. Nussinov e C.E. Yaguna, JCAP 0506, 012 (2005).

[52] C. Kaufhold e F.R. Klinkhamer, Nucl. Phys. B 734, 1 (2006).

[53] F.A. Brito, E. Passos e P.V. Santos, Europhys. Lett. 95, 51001 (2011).

[54] J. Furtado e T. Mariz, Phys. Rev. D 89, 025021 (2014).

[55] Y.M.P. Gomes e J.T. Guaitolini Junior, arXiv:1810.05202 (2018).

[56] G. Jarlskog, L. Joensson, S. Pruenster, H.D. Schulz, H.J. Willutzki e G.G. Winter, Phys. Rev. D 8, 3813(1973).

[57] A.I. Milstein e M. Schumacher, Phys. Rept. 243, 183 (1994).

[58] S.L. Adler, Annals Phys. 67, 599 (1971).

[59] S.L. Adler, C. Schubert, Phys. Rev. Lett. 77, 1695 (1996).

[60] D. d'Enterria e G.G. da Silveira, Phys. Rev. Lett. 111, 080405 (2013).

[61] J.L. Hewett, F.J. Petriello e T.G. Rizzo, Phys. Rev. D 64, 075012 (2001).

[62] V. Fock, Phys. Z. Sowjetunion 12, 404 (1937).
[63] E.C.G. Stueckelberg, Helv. Phys. Acta 15, 23 (1942).

[64] R.P. Feynman, Phys. Rev. 76, 749 (1949).

[65] R.P. Feynman, Phys. Rev. 80, 440 (1950).

[66] R.P. Feynman, Phys. Rev. 84, 108 (1951).

[67] J.S. Schwinger, Phys. Rev. 82, 664 (1951).

[68] J.M. Chung e B.K. Chung, Phys. Rev. D 63105015 (2001).

[69] P.W. Higgs, Phys. Rev. Lett. 13, 508 (1964).

[70] P.W. Anderson, Science, 177, 4047 (1972).

[71] L.D. Landau, Phys. Z. Sowjetunion, 1126 (1937).

[72] V.A. Kostelecky e S. Samuel, Phys. Rev. D 39, 683 (1989).

[73] D. Colladay e V.A. Kostelecky, Phys. Rev. D 55, 6760 (1997).

[74] D. Colladay e V.A. Kostelecky, Phys. Rev. D 58, 116002 (1998).

[75] V.A. Kostelecky, Phys. Rev. D 69, 105009 (2004).

[76] V.A. Kostelecky e R. Potting, Nucl. Phys. B 359, 545 (1991).

[77] H. Belich, T. Costa-Soares, M.A. Santos e M.T.D. Orlando, Rev. Bras. Ens. Fís. 291 (2007).

[78] V.A. Kostelecky e S. Samuel, Phys. Rev. D 40, 1886 (1989).

[79] V.A. Kostelecky e S. Samuel, Phys. Rev. Lett. 63, 224 (1989).

[80] V.A. Kostelecky e R. Potting, Phys. Lett. B 381, 89 (1996).

[81] S.M. Carroll, J.A. Harvey, V.A. Kostelecky, C.D. Lane e T. Okamoto, Phys. Rev. Lett. 87, 141601 (2001).

[82] Z. Guralnik, R. Jackiw, S.Y. Pi e A.P. Polychronakos, Phys. Lett. B 517, 450 (2001).

[83] A. Anisimov, T. Banks, M. Dine e M. Graesser, Phys. Rev. D 65, 085032 (2002).

[84] C.E. Carlson, C.D. Carone e R.F. Lebed, Phys. Lett. B 518, 201 (2001).

[85] S.M. Carroll, G.B. Field e R. Jackiw, Phys. Rev. D 41 1231 (1990).

[86] V.A. Kostelecky, C.D. Lane e A.G.M. Pickering, Phys. Rev. D 65, 056006 (2002).

[87] L.H.C. Borges, A.G. Dias, A.F. Ferrari, J.R. Nascimento e A.Y. Petrov, Phys. Lett. B 756332 (2016).

[88] R.C. Myers e M. Pospelov, Phys. Rev. Lett. 90211601 (2003).

[89] V.A. Kostelecky e M. Mewes, Phys. Rev. D 80015020 (2009).

[90] A. Kostelecky e M. Mewes, Phys. Rev. D 85096005 (2012).

[91] R. Jackiw e V.A. Kostelecky, Phys. Rev. Lett. 823572 (1999).

[92] P. Adamson, C. Andreopoulos, K.E. Arms, R. Armstrong, D.J. Auty, D.S. Ayres, B. Baller, G. Barr, W.L. Barrett, B.R. Becker et al., Phys. Rev. Lett. 101, 151601 (2008).

[93] P. Adamson, D.J. Auty, D.S. Ayres, C. Backhouse, G. Barr, W.L. Barrett, M. Bishai, A. Blake, G.J. Bock, D.J. Boehnlein et al., Phys. Rev. Lett. 105, 151601 (2010).

[94] MiniBooNE Collaboration, A.A. Aguilar-Arevalo, C.E. Anderson, A.O. Bazarko, S.J. Brice, B.C. Brown, L. Bugel, J. Cao, L. Coney, J.M. Conrad et al., Physics Letters B 718, 1303 (2013).

[95] P. Massarotti, Nuovo Cim. B 123, 840 (2008).

[96] A. Di Domenico e KLOE Collaboration, J. Phys. Conf. Ser. 171, 012008 (2009). 
[97] V.A. Kostelecky e C.D. Lane, Phys Rev. D 60116010 (1999).

[98] R. Bluhm, V.A. Kostelecký, C.D. Lane e N. Russell, Phys. Rev. Lett. 88090801 (2002).

[99] R. Bluhm, V.A. Kostelecký, C.D. Lane e N. Russell, Phys. Rev. D 68125008 (2003).

[100] H. Dehmelt, R. Mittleman, R.S. Van Dyck, Jr. e P. Schwinberg, Phys. Rev. Lett. 83, 4694 (1999).

[101] R.K. Mittleman, H.G. Dehmelt, I.I. Ioannou e N. Russell, Phys. Rev. Lett. 83, 2116 (1999).

[102] G. Gabrielse, A. Khabbaz, D.S. Hall, C. Heimann, H. Kalinowsky e W. Jhe, Phys. Rev. Lett. 82, 3198 (1999).

[103] R. Bluhm, N. Russell e A. Kostelecky, Phys. Rev. Lett. 82, 2254 (1999).

[104] V.W. Hughes, M. Grosse Perdekamp, D. Kawall e W. Liu, Phys. Rev. Lett. 87, 111804 (2001).

[105] R. Bluhm, V.A. Kostelecky e C.D. Lane, Phys. Rev. Lett. 84, 1098 (2000).

[106] H. Muller, P.L. Stanwix, M.E. Tobar, E. Ivanov, P. Wolf, S. Herrmann, A. Senger e E. Kovalchuk, Phys. Rev. Lett. 99, 050401 (2007).

[107] S.Reinhardt, G. Saathoff, H. Buhr, L.A. Carlson, A. Wolf, D. Schwalm, S. Karpuk, C. Novotny, G. Huber e M. Zimmermann, Nature Phys. 3, 861 (2007).

[108] S.R. Parker, M. Mewes, P.L. Stanwix e M.E. Tobar, Phys. Rev. Lett. 106, 180401 (2011).

[109] F. Baynes, A. Luiten e M. Tobar, Phys. Rev. D 84, 081101 (2011).

[110] V.A. Kostelecky e N. Russell, Rev. Mod. Phys. 83, 11 (2011).

[111] M. Aaboud, G. Aad, B. Abbott, J. Abdallah, O. Abdinov, B. Abeloos, S.H. Abidi, O.S. AbouZeid, N.L. Abraham, H. Abramowicz et al., Nature Phys. 13852 (2017).

[112] E. Fermi, Nuovo Cim. 2143 (1925).

[113] D. Hanneke, S. Fogwell e G. Gabrielse, Phys. Rev. Lett. 100120801 (2008).

[114] T. Takahashi, G. An, Y. Chen, W. Chou, Y. Huang, W. Liu, W. Lu, J. Lv, G. Pei, S. Pei et al., arXiv:1807.00101 (2018).

[115] B. King e T. Heinzl, High Power Laser Science and Engineering, 4, e5 (2016).

[116] S.Z. Akhmadaliev, G.Y. Kezerashvili, S.G. Klimenko, R.N. Lee, V.M. Malyshev, A.L. Maslennikov, A.M. Milov, A.I. Milstein, N. Yu. Muchnoi, A.I. Naumenkov et al., Phys. Rev. Lett. 89061802 (2002).

[117] C. Itzykson e J.B. Zuber, Quantum Field Theory (McGraw-Hill, Nova York, 1978), 1 ed.

[118] W. Greiner e J. Reinhardt, Field Quantization (Springer, Berlim, 1996), 1 ed. 